\title{
Study of the combined treatment of lung cancer using gene-loaded immunomagnetic albumin nanospheres in vitro and in vivo
}

This article was published in the following Dove Press journal:

International Journal of Nanomedicine

15 March 2016

Number of times this article has been viewed

\author{
Hao Zhang' \\ Chen Liang ${ }^{2}$ \\ Xinxin $\mathrm{Hou}^{3}$ \\ Ling Wang' \\ Dongsheng Zhang ${ }^{4}$ \\ 'Department of Imaging and \\ Nuclear Medicine, Medical School \\ of Southeast University, Nanjing, \\ Jiangsu, ${ }^{2}$ Department of Pathology, \\ The First Affiliated Hospital of \\ Nanjing Medical University, Nanjing, \\ Jiangsu, ${ }^{3}$ Department of Pathology, \\ Medical School of Henan Polytechnic \\ University, Jiaozuo, Henan, ${ }^{4}$ Jiangsu \\ Key Laboratory for Biomaterials \\ and Devices, Medical School, \\ Southeast University, Nanjing, \\ People's Republic of China
}

\begin{abstract}
Combination therapy for lung cancer has garnered widespread attention. Radiation therapy, gene therapy, and molecular targeted therapy for lung cancer have certain effects, but the disadvantages of these treatment methods are evident. Combining these methods can decrease their side effects and increase their curative effects. In this study, we constructed a pYr-ads-8-5HRE-cfosp-iNOS-IFNG plasmid (a gene circuit that can express IFN $\gamma$ ), which is a gene circuit, and used that plasmid together with $\mathrm{C} 225$ (cetuximab) to prepare gene-loaded immunomagnetic albumin nanospheres (IMANS). Moreover, we investigated the therapeutic effects of gene-loaded IMANS in combination with radiation therapy on human lung cancer in vitro and in vivo. The results showed that this gene circuit was successively constructed and confirmed that the expression of INF $\gamma$ was increased due to the gene circuit. Gene-loaded IMANS combined with radiation therapy demonstrated improved results in vitro and in vivo. In conclusion, gene-loaded IMANS enhanced the efficacy of combination therapy, solved problems related to gene transfer, and specifically targeted lung cancer cells.
\end{abstract}

Keywords: gene circuit, combination therapy, magnetic albumin nanospheres

\section{Introduction}

Lung cancer is a malignant tumor that displays one of the highest incidence and mortality rates, and these rates are significantly increasing. Lung cancer has become one of the greatest hazards to human health and life. According to its pathological and clinical characteristics, lung cancer can be classified into two major categories: small cell-lung cancer (SCLC) and non-small-cell lung cancer (NSCLC). ${ }^{1}$ The primary treatment strategy for lung cancer is surgery. Because the early symptoms of lung cancer are difficult to detect, from $70 \%$ to $80 \%$ of lung cancer cases were identified at an advanced or metastatic stage. Most of the lung cancer patients choose only chemotherapy or radiotherapy. However, due to cancer metastasis, multidrug resistance and other factors such as the survival rates of NSCLC and SCLC patients remain below $10 \%$ and 3\%, respectively. Searching for new strategies for lung cancer treatment has become a research focus. ${ }^{2-4}$ Due to the rapid development of tumor immunology, molecular biology, bioinformatics, and other disciplines, gene diagnosis, gene therapy, and targeted therapy for tumors have gradually become possible. More importantly, these advances have provided novel approaches for the treatment of lung cancer.

Gene therapy refers to the transfer of selected genes into the body of a patient to correct an aberrancy in gene expression via molecular biology techniques as a treatment for a disease. Gene therapy is primarily used to treat diseases that cause serious damage to human health, including malignant tumors, infectious diseases, genetic diseases, and

Correspondence: Dongsheng Zhang and Devices, Medical School of Southeast University, No 87 Dingjiaqiao Road,

Republic of China

Email zdszds 1222@163.com (c) (1) (5) 2016 Zhang et al. This work is published and licensed by Dove Medical Press Limited. The full terms of this license are available at https://www.dovepress.com/terms.php
and incorporate the Creative Commons Attribution - Non Commercial (unported, v3.0) License (http://creativecommons.org/licenses/by-nd/3.0/). By accessing the work you hereby accept the Terms. Non-commercial uses of the work are permitted without any further permission from Dove Medical Press Limited, provided the work is properly attributed. For permission
her for commercial use of this work, please see paragraphs 4.2 and 5 of our Terms (https://www.dovepress.com/terms.php). 
cardiovascular diseases. ${ }^{5}$ As a new clinical therapy with high specificity and good targeting, gene therapy for lung cancer has attracted increasing attention. ${ }^{6}$ Gene immunotherapy that can interact with the body's immune function is becoming an area of focus of lung cancer gene therapy. Local expression of immune-related genes in tumor cells could increase the immunogenicity of the tumor or stimulate and enhance the body's antitumor immune responses. After tumor cells are killed, the initial triggering activity is halted. IFN $\gamma$ exerts direct antitumor effects by inhibiting tumor cell proliferation, inducing differentiation, and promoting apoptosis ${ }^{7,8}$ and exerts indirect antitumor effects by activating macrophages and natural killer cells, inducing the expression of major histocompatibility complex class I and class II molecules, enhancing the T-cell-mediated death of tumor cells and inhibiting tumor angiogenesis. ${ }^{9}$ The transfer of the IFN $\gamma$ gene into tumor cells, which then abundantly expresses IFN $\gamma$, resulting in their death, has been the basis of many research prospects.

In radio-gene therapy, therapeutic genes are fused to a radiation-induced regulatory sequence. When the tumor is irradiated by radiotherapy/radionuclide, gene expression is induced, and the tumor is attacked by both the radiation and the induced gene. On the one hand, the equivalent radiation dose can be reduced to decrease damage to normal tissues; on the other hand, the localization of antitumor gene expression can be achieved via local irradiation. ${ }^{10}$

However, this method displays the same disadvantages as conventional gene therapy, such as specificity, safety, low efficiency and short duration of gene expression, low cytotoxic effect, lack of effective control measures after gene transfer, and other issues. Moreover, the prevalent hypoxic environment of solid tumors decreases radiation-induced promoter activity, thereby negatively affecting the efficacy and application of this therapy. ${ }^{11,12}$

Gene circuit technology represents a new concept for the development of tumor radio-gene therapies. A gene circuit refers to the network which consists of specific genes has the signal processing functions to regulate the target gene like the physical circuit. The characteristic of a gene circuit is that different genes exhibit feedback regulation, in which upstream genes induce the expression of downstream genes and the protein products of downstream genes regulate the expression of upstream genes, such that the genes form a circuit. Constructing a physiological genetic circuit and transferring it into cells may regulate and amplify target gene expression. ${ }^{13,14}$ Gene circuit design must fully consider the inhibitory effects of the hypoxic tumor environment on radiation-induced promoter activity. The hypoxia-response element (HRE) is a hypoxia-sensitive enhancer sequence. Studies have shown that inserting an HRE sequence upstream of a gene promoter enhances the transcriptional activity of the promoter in a hypoxic environment and that the number of HREs influences the sensitivity of the promotor to hypoxia. ${ }^{15,16}$

However, naked DNA is not stable, as it is easily degraded by nucleases. ${ }^{17}$ Therefore, gene transfer is mediated by a gene vector. Currently, the most common gene vectors are viral vectors and nonviral vectors. ${ }^{18}$ The viral vector system is by far the most effective method of gene transfer. Two-thirds of clinical trials performing tumor gene therapy used viral vectors. ${ }^{19,20}$ However, the limitations of viral vectors, such as the lack of efficient and targeted carrier systems, the difficulty in controlling genes in the body, and, especially, safety ${ }^{21,22}$ have restricted the clinical application of viral vectors for gene therapy. Although using a nonviral vector system avoids significant potential safety hazards, the transduction efficiency of nonviral vector systems has always been less than that of viral vector systems. Therefore, how to resolve the bottleneck of gene transfer has become imperative in the field of gene therapy.

With the development of nanotechnology, research on nanoparticles as gene transfer vectors has garnered widespread attention. Nanoparticle vectors represent a new system of polymer transport. Because of their small size, nanoparticles easily carry a target gene to cells, and the gene easily diffuses into cells. A variety of nanoparticle gene transfer vectors have been developed, such as gold nanoparticles, nanomagnetic particles, and albumin nanoparticles (ANPs). Studies have shown that these nanoparticle vectors are easy to prepare, display adequate DNA adsorption and high transfection efficiency, and exhibit acceptable biocompatibility. Nanoparticle vectors conjugated to specific targeting molecules (such as monoclonal antibodies or specific ligands) that can bind to cell surface receptors can provide safe and effective targeted therapy. ${ }^{23-30}$ Nanomagnetic particle vector technology uses magnetic nanoparticles as a gene vector to deliver therapeutic genes to cells; using this method, the nanoparticles release their contents in the presence of a magnetic field to achieve safe and effective targeted therapy. ${ }^{31,32}$ This technology can help to accelerate transfection while increasing the transfection efficiency. Therefore, magnetic nanoparticles have rapidly been developed as gene vectors. Many scholars have performed intensive research on this topic. 
Magnetic albumin nanospheres (MANS) are albumin nanospheres that are wrapped with magnetic nanoparticles..$^{33}$ As a carrier, albumin nanospheres can reduce the drug release rate, avoid drug inactivation during the transfer process, and increase the biocompatibility of nanomaterials. Applying a magnetic field outside the tumor site concentrates the drug at the tumor site. As a result, the antitumor effect of the drug is enhanced and the damage to normal tissue is reduced. ${ }^{34,35}$

Molecular targeted therapy for tumors is a completely new model of biological treatment. This form of therapy targets factors that may cause cells to become malignant, such as cell signaling pathways, proto-oncogenes, tumor suppressor genes, cytokines and their receptors, tumor angiogenesis-related factors, and cell death signaling-related genes. Molecular targeted therapy reverses malignant biological behaviors at the molecular level; as a consequence, tumor cell growth is inhibited and the tumor even completely disappears. The epidermal growth factor receptor (EGFR) is expressed in various malignant tumor cells that arise from the epidermis, such as NSCLC, ${ }^{36}$ breast cancer, colorectal cancer, ${ }^{37}$ gastric cancer, and prostate cancer. Therapies targeting EGFR are extensively administered in the field of lung cancer. Such therapies include 1) monoclonal antibodies against the extracellular domain of EGFR; 2) small molecule kinase inhibitors that act on the EGFR kinase domain; 3) antisense oligonucleotides that block EGFR mRNA translation; 4) cytotoxic agents that can recognize EGFR, such as cell death factors and radioactive particles, selectively killing tumor cells displaying elevated EGFR expression; and 5) blockers of EGFR dimerization/ oligomerization, thereby inhibiting the activation of EGFR..$^{38}$ At present, anti-EGFR monoclonal antibodies and small molecule tyrosine kinase inhibitors are most commonly used in clinical settings. Cetuximab (C225), marketed as Erbitux, is a chimeric human/mouse immunoglobulin G1 antibody that can specially bind to the extracellular domain of human EGFR. C225 can competitively interact with the extracellular ligand binding site of EGFR, thereby inhibiting the activation of the tyrosine kinase activity of EGFR. As a result, the signal transduction pathways associated with tumor cell proliferation are blocked, and this event inhibits cell proliferation and induces apoptosis. In addition, studies have shown that antiEGFR monoclonal antibodies can enhance the sensitivity of chemotherapy and radiotherapy. ${ }^{39}$

In this study, we hypothesized that inserting an HRE upstream of the radiation-induced promoter of c-Fos would form the radiation promoter that exhibits enhanced expression in a hypoxic environment. Thus, we constructed the pYr-ads-8-5HRE-cfosp-iNOS-IFNG eukaryotic expression plasmid, which expresses a gene circuit. The properties of this plasmid are that the c-Fos promoter induces the expression of the inducible nitric oxide synthase (NOS, iNOS) and $I F N \gamma$ genes; then, NO, which is a product of iNOS activity, induces the c-Fos promoter via a feedback loop. As a gene carrier, MANS was coupled to $\mathrm{C} 225$ to construct gene-loaded immunomagnetic albumin nanospheres (IMANS). In cancer treatment, the gene-loaded IMANS were targeted to lung cancer tissues via intravenous injection. We explored the efficacy of this novel combination therapy consisting of radiation therapy, immune-related gene therapy, and molecular targeted therapy for lung cancer.

\section{Materials and methods Main materials}

Roswell Park Memorial Institute (RPMI)-1640 medium and fetal bovine serum were obtained from Thermo Fisher Scientific (Waltham, MA, USA). Cell Counting Kit-8 (CCK8) was obtained from Dojindo (Kumamoto, Japan). Agarose, bovine serum albumin, dimethyl sulfoxide, trypsin, $\mathrm{N}$-succinimidyl-3-(2-pyridyldithio)propionate (SPDP), and DL-dithiothreitol were supplied by Sigma-Aldrich Co. (St Louis, MO, USA). The human lung cancer GLC-82 cell line was provided by the Institute of Biochemistry and Cell Biology of the Shanghai Institute of Biological Sciences at the Chinese Academy of Sciences.

\section{Construction of the 5HRE-cfosp-iNOS- IFNG (IFN $\gamma$ ) plasmid}

The 796 bp human c-Fos gene promoter sequence, which contained the core promoter region, was synthesized by Yingrun Corporation (Changsha, People's Republic of China). This sequence was inserted into the MluI-EcoRI site of the $\mathrm{pYr}$ adshuttle-8 vector (Yingrun Corporation), replacing the CMV promoter, thereby creating the $\mathrm{pYr}$-ads-8-cfosp vector.

The 5HRE (five copies of HREs) region was excised from the pUC57-5HRE plasmid (Yingrun Corporation) via digestion with NheI and BglII. Next, this region was inserted into the NheI-BglII site of pYr-ads-8-cfosp to obtain pYr-ads-8-5HRE-cfosp recombinant plasmid. The IFNG (IFN $\gamma)$ gene sequence was excised from the pDONR223IFNG plasmid (Yingrun Corporation) and was subcloned into the pYr-ads-8-5HRE-cfosp plasmid to generate the pYr-ads-8-5HRE-cfosp-IFNG recombinant plasmid. The iNOS gene sequence was excised from the pYr-ads-1-iNOS vector (Yingrun Corporation) via digestion with $\mathrm{XbaI}$ and SalI. Then, this sequence was inserted into the Xbal-SalI site of the pYr-ads-8-5HRE-cfosp-IFNG plasmid to obtain 
pYr-ads-8-5HRE-cfosp-iNOS-IFNG plasmid. In addition, the pYr-ads-8-cfosp-iNOS-IFNG plasmid was constructed as a control in the same manner as described earlier. The sequence of all recombinant plasmids was confirmed by sequencing and digestion analyses.

\section{Preparation of gene-loaded MANS via the desolvation-cross-linking method}

PEI-Fe ${ }_{3} \mathrm{O}_{4}$ magnetic nanoparticles (stored at our lab) and the pYr-ads-8-5HRE-cfosp-iNOS-IFNG or pYr-ads-8-cfospiNOS-IFNG plasmid diluted at the indicated mass ratios (30:1) were incubated for 30 minutes. ${ }^{40}$ Then, bovine serum albumin $(100 \mathrm{mg})$ was added and the mixture was stirred. The $\mathrm{pH}$ of the mixture was adjusted to 9 . Next, ethanol was added drop-wise to the solution at a rate of $1 \mathrm{~mL} / \mathrm{min}$, followed by the slow addition of $50 \mu \mathrm{L}$ of $2.5 \%$ glutaraldehyde. After stirring for 24 hours at room temperature (RT), the solution was washed three times, and the organic solvent added during the preparation was removed via high-speed centrifugation to yield gene-loaded MANS. The gene-loaded MANS that were produced included HRE-IFNG-MANS and IFNG-MANS.

\section{Preparation of gene-loaded IMANS using a heterobifunctional cross-linker}

SPDP was used to couple nanospheres to the monoclonal antibody $\mathrm{C} 225$ according to the following protocol:

First, an adequate amount of $\mathrm{C} 225$ was dissolved in $0.01 \mathrm{M}$ phosphate-buffered saline (PBS) $(\mathrm{pH}=7.4)$ and $20 \mathrm{mM}$ SPDP. Ethanol was added to the solution at a molar ratio of 1:15. After 60 minutes, the mixture was dialyzed with acetate buffer at RT to remove excess SPDP. A sufficient amount of DL-dithiothreitol was added to the pyridine dithiobis-conjugated monoclonal antibody (McAb-PDP-SH) solution obtained in the previous step, and the mixture was gently stirred for 30 minutes at RT. Then, the reactant was dialyzed with PBS to remove excess DL-dithiothreitol and to obtain a thiol antibody solution.

Second, an appropriate amount of gene-loaded MANS was diluted in acetate buffer and $50 \mu \mathrm{L}$ of ethanol solution containing $20 \mathrm{mM}$ SPDP was added with stirring; this was then centrifuged to remove excess SPDP.

Third, the activated gene-loaded MANS and McAbPDP-SH mixture was incubated and shaken at $4{ }^{\circ} \mathrm{C}$ for 15 hours. After washing several times via centrifugation, gene-loaded IMANS were obtained. The gene-loaded IMANS that were produced included HRE-IFNG-IMANS and IFNG-IMANS.

Transmission electron microscopy and dynamic light scattering (DLS) were used to characterize the gene-loaded
IMANS. To assess the stability of the gene-loaded IMANS, the hydrated size of the gene-loaded IMANS in PBS was analyzed by DLS within 24 hours, and the particle size was measured for 32 days with storage at $4^{\circ} \mathrm{C}$ and ambient humidity.

\section{Assessment of the expression of IFNG via quantitative PCR}

Cell culture

Human lung cancer GLC-82 cell lines were maintained in RPMI-1640 medium supplemented with $10 \%$ fetal calf serum and incubated in a humidified $5 \% \mathrm{CO}_{2}$ atmosphere at $37^{\circ} \mathrm{C}$ (normoxia). Hypoxia was induced by culturing the cells in an incubator containing $1 \% \mathrm{O}_{2}, 5 \% \mathrm{CO}_{2}$, and $94 \%$ $\mathrm{N}_{2}$ at $37^{\circ} \mathrm{C}$.

\section{Cell transfection with gene-loaded IMANS}

GLC-82 cells were seeded in six-well plates at an initial density of $3 \times 10^{5}$ cells/well in $2 \mathrm{~mL}$ of growth medium. After incubation for 24 hours and once the cells had reached $80 \%$ confluence, the medium was replaced with $1.5 \mathrm{~mL}$ of serum-free media and $500 \mu \mathrm{L}$ of empty IMANS, IFNG-IMANS, or HRE-IFNG-IMANS, and the cells were incubated for an additional 4 hours. Then, the medium was replaced with fresh medium containing serum. After incubation for 24 hours, the IFNG-transfected GLC-82 (GLC-82/ IFNG) cells were exposed to $2 \mathrm{~Gy}$ of X-ray under an X-ray biological irradiator (RS2000Pro; Rad Source Technologies, Inc., Suwanee, GA, USA). The negative group used normal GLC-82 cells. Then, the cells were incubated for an additional 48 hours in a hypoxic environment. Then, total RNA was extracted from the GLC-82/IFNG cells. As a blank control, total RNA was extracted from untreated GLC-82 cells. GAPDH was used as an internal reference gene. Then, we quantitatively analyzed the gene expression levels of IFN $\gamma$ via quantitative polymerase chain reaction PCR (qPCR). All reagents and materials required to extract RNA were RNase-free or were soaked in diethypyrocarbonate water. The following primer sequences were synthesized by Nan Jing Jin Sirui Company (Nanjing, Jiangsu, People's Republic of China):

\section{Human GAPDH primer (132 bp)}

Sense primer: 5'-TGGTATCGTGGAAGGACTCA-3' Antisense primer: 5'-CCAGTAGAGGCAGGGATGAT-3'

Human IFN $\gamma$ primer (142 bp)

Sense primer: 5'-AGATGACCAGAGCATCCAAA-3'

Antisense primer: 5'-TTTGCGTTGGACATTCAAGT-3' 


\section{Evaluation of GLC-82 cell-specific cytotoxicity of gene-loaded IMANS (HRE-IFNG-IMANS) in vitro}

GLC-82 cells $\left(4 \times 10^{4}\right)$ were cultured in three 96-well plates. After incubation for 24 hours, the cells were subjected to six different conditions:

1) Negative control (1640 containing 10\% fetal calf serum)

2) Free $\mathrm{C} 225$ treatment (C225 solution)

3) Radiotherapy (2 Gy X-ray)

4) Gene therapy combined with radiotherapy (HRE-IFNGMANS+2 Gy X-ray)

5) Free $\mathrm{C} 225$ combined with radiotherapy (C225 solution +2 Gy X-ray)

6) Gene-loaded IMANS combined with radiotherapy (HREIFNG-IMANS+2 Gy X-ray).

After incubation for 24 hours, the 96-well culture plates in the radiotherapy groups were exposed to 2 Gy of X-ray radiation under an X-ray biological irradiator. All groups were further incubated. At 24,48 , and 72 hours, $10 \mu \mathrm{L}$ of CCK8 solution was added to each well, and the cells were incubated at $37^{\circ} \mathrm{C}$ for 1-4 hours. The optical density (OD) at $450 \mathrm{~nm}$ was recorded using a spectrophotometer (Infinite 50; TECAN, Männedorf, Switzerland). The following formula was used to calculate the relative inhibition rate (RIR) of cell proliferation:

$$
\text { RIR }=\left(\begin{array}{c}
\text { experimental group OD - } \\
\text { blank control group OD } \\
\text { negative control group OD - } \\
\text { blank control group OD }
\end{array}\right) \times 100 \%
$$

\section{Establishment of lung cancer xenografts in nude mice}

Healthy 4-week-old female BALC/c nude mice weighing 18-20 g were used for these experiments. The animal experiments were approved by and performed in accordance with the institutional guidelines of the Animal Care Committee of Jiangsu Province. All mice were housed in the Sterile Barrier System of the Medical School, Southeast University, People's Republic of China. Exponentially growing GLC-82 cells $\left(2 \times 10^{6}\right)$ were subcutaneously injected into the limbs of the mice to establish lung cancer xenografts. All cell and animal studies were approved by the Ethics Committee of Southeast University, Nanjing, China.

\section{Animal experiments}

When the tumor diameter reached approximately $1 \mathrm{~cm}$, the mice were divided into six groups of six mice each. These groups were consistent with the CCK8 experiment described earlier. The mice bearing xenografts were injected with normal saline (group 1), C225 solution (groups 2 and 5), HRE-IFNGMANS (group 4), or HRE-IFNG-IMANS (group 6) via the tail vein. HRE-IFNG-MANS and HRE-IFNG-IMANS contained $10 \mu \mathrm{g}$ of the pYr-ads-8-5HRE-cfosp-iNOS-IFNG plasmid. The tumors of groups 3, 4, 5, and 6 were exposed to 2 Gy of X-ray radiation under an X-ray biological irradiator every other day for a total of three sessions. The mice were killed after 5 weeks, and the mass and the volume of each tumor were measured. Tumor growth inhibition was evaluated by measuring the percentages of tumor mass and volume inhibition. Mass inhibition was calculated as (1-relative tumor mass $\times 100 \%$ ), where the relative tumor mass is the mean tumor mass of the experimental group divided by the mean tumor mass of the control group. Similarly, volume inhibition was calculated as (1-relative tumor volume $\times 100 \%)$, where the relative tumor volume is the mean tumor volume of the experimental group divided by the mean tumor volume of the control group. After killing the mice, the tumor tissues were collected and fixed with 10\% formaldehyde. Then, the tumor tissues were embedded in paraffin, stained with hematoxylin and eosin, and observed via light microscopy.

\section{Statistical analysis}

The values are shown as mean \pm standard deviation. The data were analyzed using SPSS 16.0 software (SPSS Inc., Chicago, IL, USA). A $P$-value of $<0.05$ was considered significant.

\section{Results}

\section{Characterization of the $\mathrm{p} 5 \mathrm{HRE}$-cfosp- iNOS-IFNG plasmid}

Theoretically, digestion of the p5HRE-cfosp-iNOS-IFNG construct with EcoRI would produce a small 900 bp fragment and a large $8.3 \mathrm{~K}$ fragment. As shown in Figure 1, agarose gel electrophoresis of p5HRE-cfosp-iNOS-IFNG digested with EcoRI clearly showed two bands that are approximately 900 bp and $8.3 \mathrm{~K}$ in size in lane 2 ; this result confirmed the production of the p5HRE-cfosp-iNOS-IFNG recombinant plasmid.

The corresponding sequencing file is pYr-5HRE-cfospiNOS-IFNG. ab1. The sequencing results were compared with the template sequence and this comparison (Figure 2) showed that the sequence was completely correct. Therefore, the pYr-5HRE-cfosp-iNOS-IFNG plasmid was successfully constructed.

\section{Preparation and characterization of gene- loaded IMANS}

Transmission electron microscopy analysis showed that the self-prepared gene-loaded IMANS were approximately 


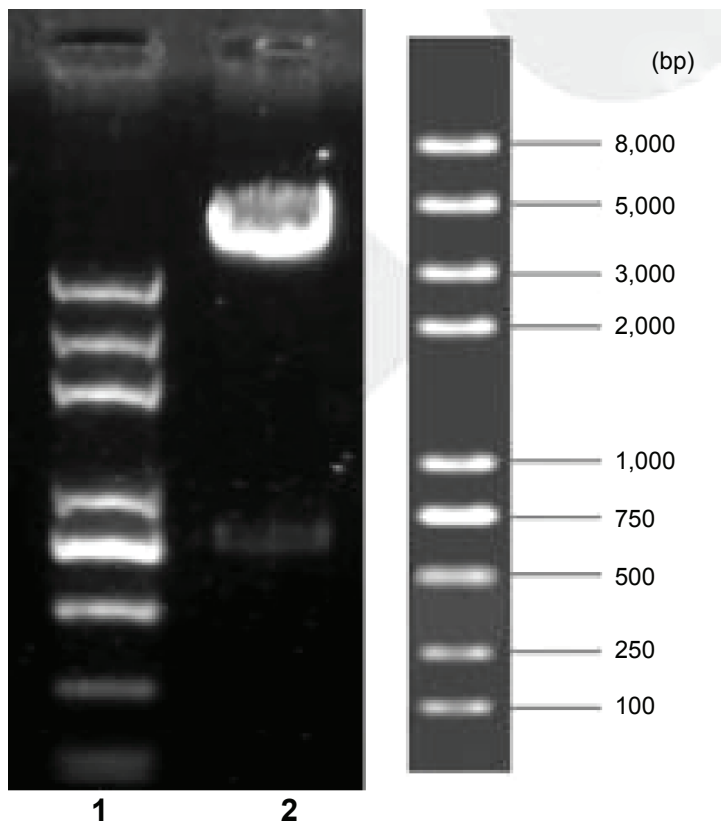

Figure I The pYr-5HRE-cfosp-iNOS-IFNG plasmid was identified via restriction enzyme digestion.

Notes: p5HRE-cfosp-iNOS-IFNG digested with EcoRI clearly showed two bands of $\sim 900 \mathrm{bp}$ and $8.3 \mathrm{~K}$ in size in lane 2. Lane I, marker; lane 2, pYr-5HRE-cfosp-iNOSIFNG plasmid digested with EcoRI.

Abbreviations: HRE, hypoxia-response element; iNOS, inducible nitric oxide synthase. spherical and uniform in size. The magnetite materials with a high electron density were fully incorporated into the cores of ANS (Figure 3A). DLS showed that the hydrated volume size of the gene-loaded IMANS particles was $134.1 \pm 2.8 \mathrm{~nm}$ and that the polydispersity index was 0.157 (Figure 3B[1]). The size measurement by number of gene-loaded IMANS was $149.7 \pm 3.1 \mathrm{~nm}$.

To assess the stability of the gene-loaded IMANS, the hydrated size of the gene-loaded IMANS was analyzed by DLS. The gene-loaded IMANS showed limited variation $(<10 \%)$ in volume size and number size after 32 days of storage in PBS at $4^{\circ} \mathrm{C}$, indicating excellent stability in aqueous medium.

\section{Transfection of the IFNG gene into GLC-82 cells using gene-loaded IMANS}

The mRNA level of IFNG in GLC-82 cells was assessed via qPCR. The GAPDH gene was used as an internal control to rule out any operational error in the PCR process and to calculate the relative expression level of IFNG. The first group was the negative control group; the second group was the empty IMANS group; the third group was the IFNG-IMANS group; and the fourth group was the HRE-IFNG-IMANS group.

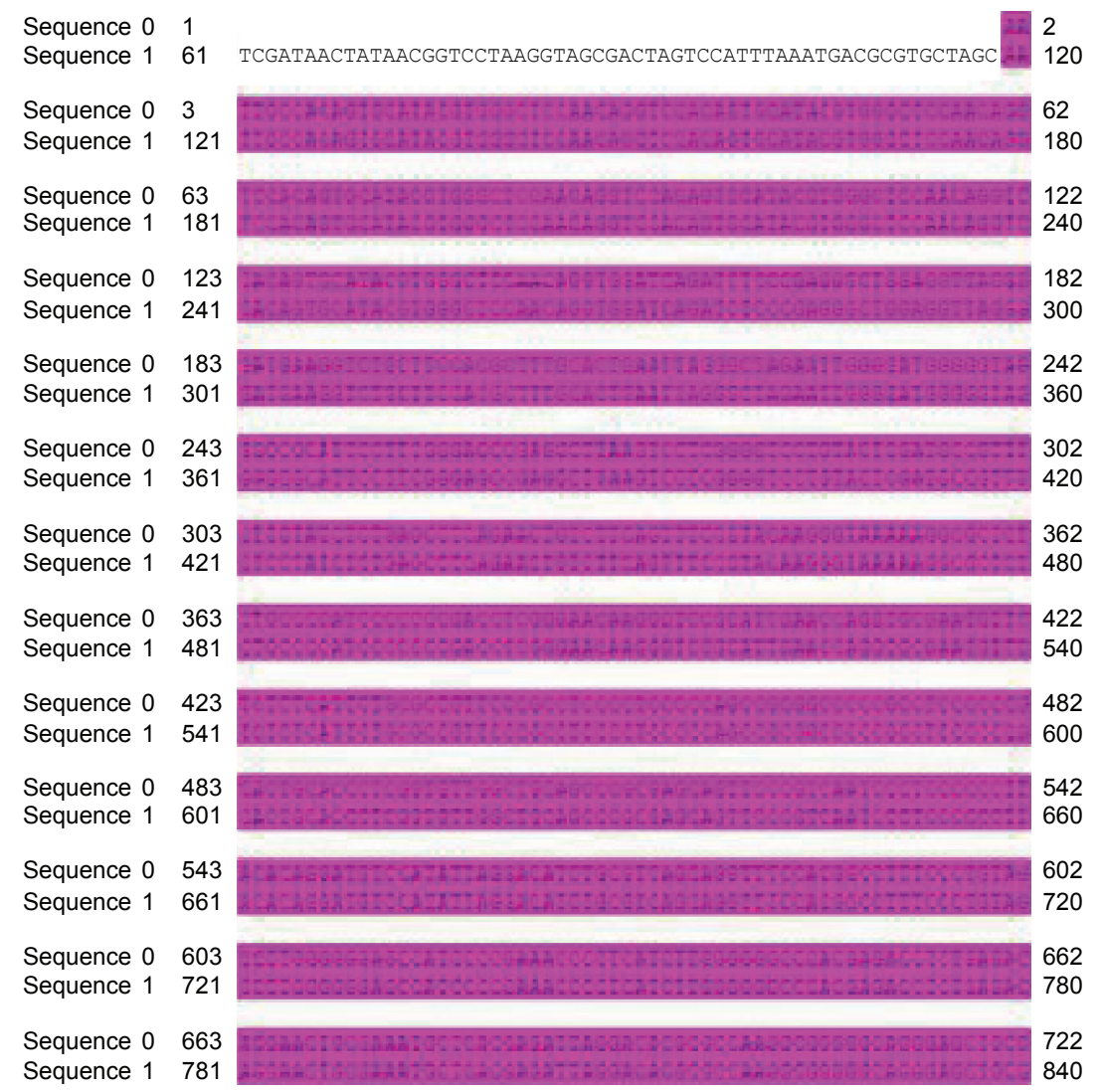

Figure 2 The sequencing results.

Notes: The sequencing results were compared with the template sequence, and this comparison showed that the sequence was completely correct. Sequence 0 , $5 \mathrm{HRE}$-cfosp template sequence; sequence I, sequence obtained from sequencing analysis. Comparison software used was Dnassit 2.0 (Dnassit, Imola, BO, Italy).

Abbreviation: HRE, hypoxia-response element. 

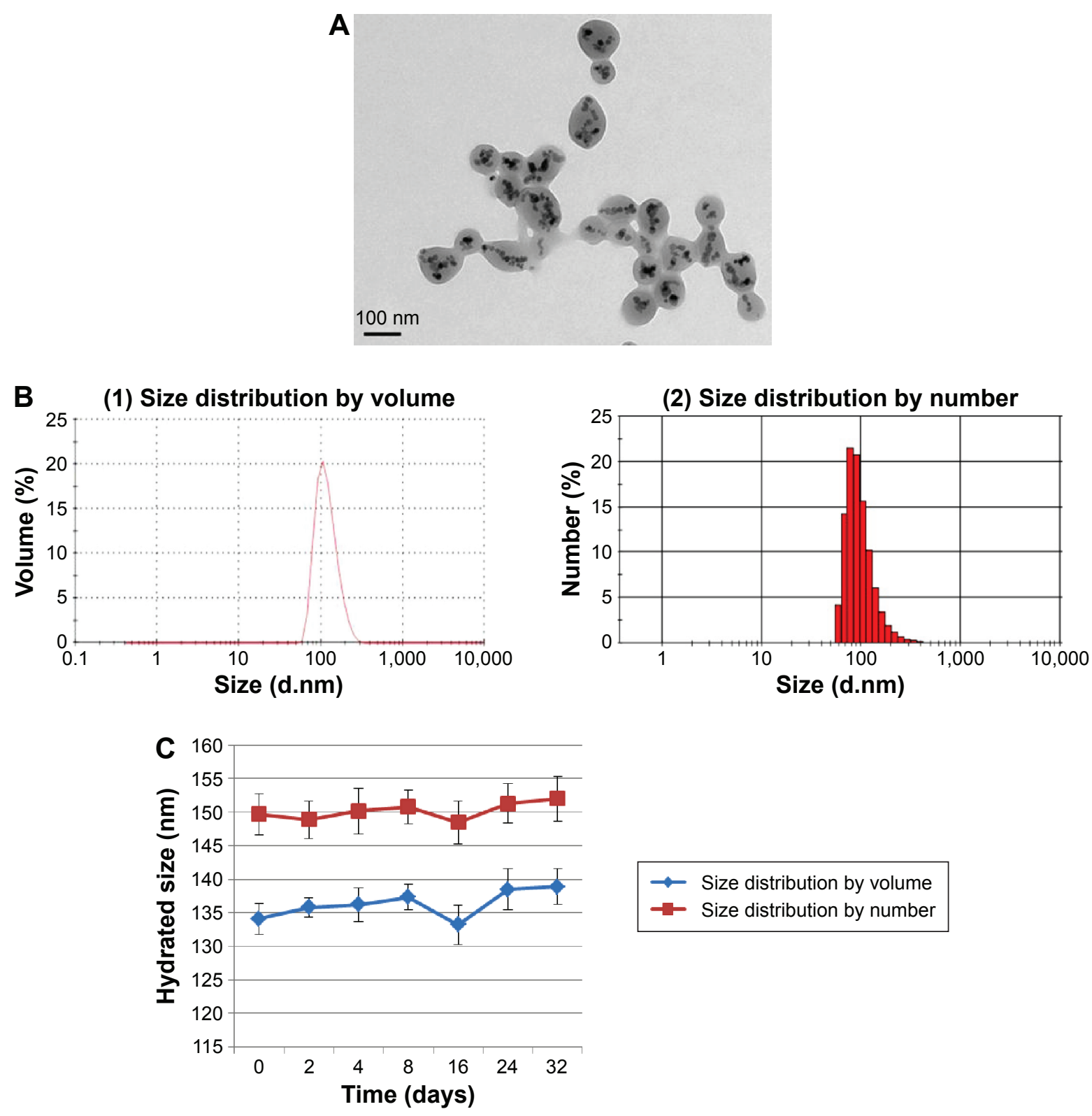

- Size distribution by number

Figure 3 The characterization of gene-loaded IMANS.

Notes: (A) TEM of gene-loaded IMANS. The self-prepared gene-loaded IMANS were approximately spherical and uniform in size. The magnetite materials with a high electron density were fully incorporated into the cores of ANS. (B) Volume size and number size of gene-loaded IMANS by DLS in PBS. (I) The size distribution of geneloaded IMANS by volume. The hydrated volume size of the gene-loaded IMANS particles was $134.1 \pm 2.8 \mathrm{~nm}$. (2) The size distribution of gene-loaded IMANS by number. The number size of gene-loaded IMANS was $149.7 \pm 3.1 \mathrm{~nm}$. (C) Hydrated size of gene-loaded IMANS by DLS in PBS. The gene-loaded IMANS showed limited variation (<I0\%) in volume size and number size after 32 days of storage in PBS at $4^{\circ} \mathrm{C}$, indicating excellent stability in aqueous medium.

Abbreviations: TEM, transmission electron microscopy; DLS, dynamic light scattering; PBS, phosphate-buffered saline; IMANS, immunomagnetic albumin nanospheres; ANS, albumin nanospheres.

As shown in Figure 4, compared with the control group, the HRE-IFNG-IMANS group and IFNG-IMANS group displayed significantly higher IFN $\gamma$ expression. But the empty IMANS group did not display any IFN $\gamma$ expression. In the hypoxic environment, the IFN $\gamma$ expression level in the HREIFNG-IMANS group was significantly higher than that in the IFNG-IMANS group $(P<0.05)$. These results suggested that the pYr-5HRE-cfosp-iNOS-IFNG plasmid was successfully transfected into GLC-82 cells using gene-loaded IMANS and that the transgene was stably expressed at the mRNA level.

\section{Evaluation of the GLC-82 cell-specific cytotoxicity of gene-loaded IMANS (HRE-IFNG-IMANS) in vitro}

The RIR of cell proliferation in the six groups after 24, 48, and 72 hours is shown in Figure 5. The RIR significantly differed between the experimental and negative control groups $(P<0.05)$. Reduced cell proliferation was observed in every treatment group. Compared with the single therapy groups (groups 2 and 3) and the other two therapy groups 


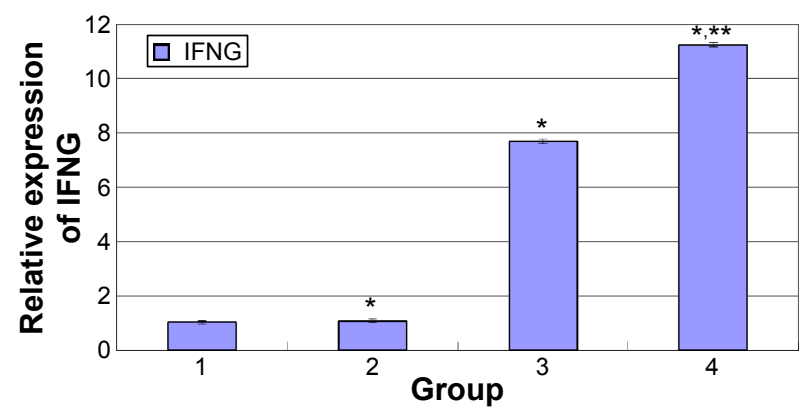

Figure 4 The relative expression quantity of IFN $\gamma$.

Notes: Group I is the negative control group, group 2 is the empty IMANS group, group 3 is the IFNG-IMANS group, and group 4 is the HRE-IFNG-IMANS group. The HRE-IFNG-IMANS group displayed the highest IFN $\gamma$ expression in the hypoxic environment. *Comparison between the experimental group and the negative control group, $P<0.05$; **comparison between hypoxia group and normoxia group, $P<0.05$.

Abbreviations: HRE, hypoxia-response element; IMANS, immunomagnetic albumin nanospheres.

(groups 4 and 5), the gene-loaded IMANS combined with radiotherapy group significantly further inhibited the proliferation of GLC-82 cells $(P<0.05)$.

\section{Effect of gene-loaded IMANS combined with radiotherapy against lung cancer in vivo}

As shown in Table 1, treatment with the gene-loaded IMANS composite nano-system combined with radiotherapy greatly inhibited the growth of tumors in nude mice, and this effect was much stronger than that of radiation alone, C225 alone, or any other combination therapy. The percentages of tumor volume inhibition and mass inhibition in group 6 were $85.72 \%$ and $82.54 \%$, respectively; these values were significantly higher than the respective values of $34.33 \%$ and $36.51 \%$ in group $2,40.89 \%$ and $42.06 \%$ in group 3 , $65.64 \%$ and $64.29 \%$ in group 4 , and $72.57 \%$ and $70.63 \%$ in group 5.

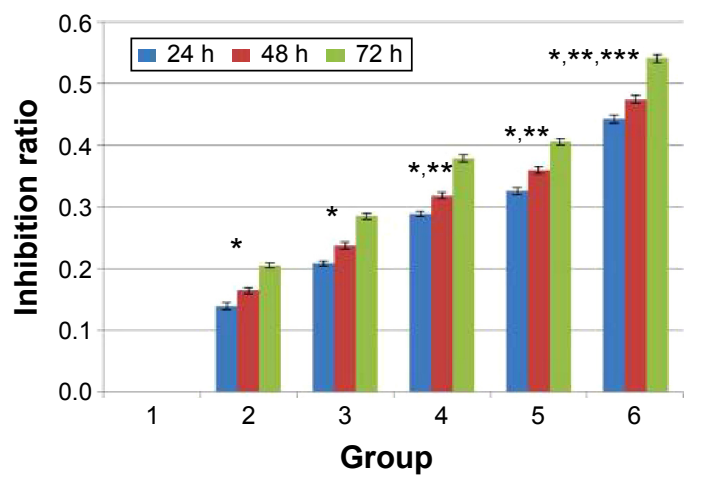

Figure 5 The results of the CCK8 assay.

Notes: The RIR significantly differed between the experimental and negative control groups, and the gene-loaded IMANS combined with radiotherapy group significantly further inhibited the proliferation of GLC- 82 cells; *comparison between the experimental group and the negative control group, $P<0.05$; ** compared to groups 2 and $3, P<0.05$; ****ompared to groups 4 and $5, P<0.05$.

Abbreviations: CCK8, cell counting kit-8; RIR, relative inhibition rate; IMANS, immunomagnetic albumin nanospheres; h, hours.
Histological analysis of the tumor tissues is shown in Figure 6. In the negative control group, the tumor cells were irregular and exhibited varying sizes, and the cell nuclei were large and hyperchromatic. Furthermore, several cells contained two or more nuclei. The tumor tissues of the treatment groups displayed different degrees of necrosis, and we observed the infiltration of inflammatory cells surrounding the areas of partial necrosis. Tumor necrosis was the most extensive in group 6 (the necrotic areas are stained pink), in which massive necrosis was observed as demonstrated by the disintegration of tumor cells and the disappearance of nuclei.

\section{Discussion}

Few genes are both induced by radiation and perform antitumor functions. Radiation-induced genes, such as $c-F o s$, c-Jun, Egr-1, and $N F \kappa B$, have been demonstrated to play an important role in cell proliferation, apoptosis, and necrosis. The expression of these genes in cells increases in response to exposure to radiation. The $c-F o s$ gene, a carcinomarelated gene that encodes a nuclear protein, is expressed in a variety of malignant tumors. ${ }^{41}$ Furthermore, the $c$-Fos gene is an immediate early gene that plays an important role in cell adaptation to stress-induced damage. The $c$-Fos gene promoter contains an SRE and other transcription-regulating sequences, which are radiation-induced sequences that induce the expression of downstream genes in response to stimulation with radiation. ${ }^{42}$ Antitumor genes that lack the ability to be induced by radiation can interact with the $c$-Fos gene to obtain radiation inducibility. In recent years, another radiation-induced promoter that has been investigated is Egr-1. Since Weichselbaum ${ }^{43}$ linked Egr-1 to the cDNA of TNF $\alpha$ and successfully induced the expression of TNF $\alpha$ by applying radiation to tumor cells, radiation-induced gene 
Table I Volume and mass inhibition of lung cancer in nude mice after different treatments

\begin{tabular}{|c|c|c|c|c|}
\hline Group & $\begin{array}{l}\text { Tumor volume }\left(\mathrm{mm}^{3}\right) \\
(\bar{X} \pm \mathbf{S}, \mathrm{n}=5)\end{array}$ & $\begin{array}{l}\text { Tumor mass (g) } \\
(\bar{X} \pm S, n=5)\end{array}$ & Volume inhibition (\%) & Mass inhibition (\%) \\
\hline Negative control group & $1,229.86 \pm 130.40$ & $1.26 \pm 0.023$ & - & - \\
\hline Free C225 therapy group & $807.63 \pm 135.87$ & $0.80 \pm 0.013$ & $34.33^{\mathrm{a}}$ & $36.5 \mathrm{I}^{\mathrm{a}}$ \\
\hline Radiotherapy group & $726.94 \pm 157.63$ & $0.73 \pm 0.031$ & $40.89^{a}$ & $42.06^{\mathrm{a}}$ \\
\hline $\begin{array}{l}\text { Gene therapy combined } \\
\text { with radiotherapy group }\end{array}$ & $422.56 \pm 121.89$ & $0.45 \pm 0.021$ & $65.64^{\mathrm{a}, \mathrm{b}}$ & $64.29^{\mathrm{a}, \mathrm{b}}$ \\
\hline $\begin{array}{l}\text { C225 combined with } \\
\text { radiotherapy group }\end{array}$ & $337.41 \pm 152.46$ & $0.37 \pm 0.015$ & $72.57^{\mathrm{a}, \mathrm{b}}$ & $70.63^{\mathrm{a}, \mathrm{b}}$ \\
\hline $\begin{array}{l}\text { GIMANS combined with } \\
\text { radiotherapy group }\end{array}$ & $175.65 \pm \mid 25.84$ & $0.22 \pm 0.025$ & $85.72^{\mathrm{a}, \mathrm{b}, \mathrm{c}}$ & $82.54^{\mathrm{a}, \mathrm{b}, \mathrm{c}}$ \\
\hline
\end{tabular}

Notes: Treatment with the gene-loaded IMANS combined with radiotherapy greatly inhibited the growth of tumors in nude mice, and this effect was much stronger than that of radiation alone, $\mathrm{C} 225$ alone, or any other combination therapy. ${ }^{a} P<0.05$ compared to the blank control group. ${ }^{b} P<0.05$ compared to groups 2 and 3 . ${ }^{c} P<0.05$ compared to groups 4 and 5 . These measurements were obtained after treatment for 5 weeks.

Abbreviations: GIMANS, gene-loaded immunomagnetic albumin nanospheres; C225, cetuximab; IMANS, immunomagnetic albumin nanospheres; S, standard deviation.

therapy has been used in the research of hepatoma, lung cancer, breast cancer, and other malignant tumors. Some scholars linked Egr-1 to a hematopoietic colony-stimulating factor and transferred the fused gene into hematopoietic cells to stimulate bone marrow hematopoiesis. As a result, bone marrow suppression was alleviated during the process of radiotherapy. ${ }^{44}$

Gene circuit technology provides a new strategy for radiotherapy and serves as an effective method to resolve the difficulties in gene therapy, such as the ineffectiveness and short duration of gene expression. In our study, we used the $i N O S$ gene to construct the gene circuit because the expression product of iNOS is NO. NOS catalyzes the production of $\mathrm{NO}$ in the body from $\mathrm{L}$-arginine. $\mathrm{NO}$ has been identified as an important second messenger with the following properties. ${ }^{45,46} 1$ ) NO is a fat-soluble small molecule in a gaseous state that can freely diffuse between cells. 2) Because its half-life is short, its range of activity is limited and it rarely induces systemic reactions. 3) NO can rapidly activate the $c-F o s$ gene promoter via the cGMP/GK pathway to induce downstream gene expression. Worthington et al fused the radiation-induced WAF1 promoter to the $i N O S$ gene and found that the expression of iNOS was clearly increased in response to irradiation. Subsequently, Worthington et al constructed the E9 promoter (containing nine CarG elements) and fused this promoter to the $i N O S$ gene to form the $\mathrm{pE} 9 \mathrm{iNOS}$ plasmid. As a result, iNOS expression and NO production were induced by irradiation; in turn, NO activated the E9 promoter. Therefore, a positive feedback gene circuit was formed. ${ }^{13-15,47,48}$

Some scholars have fused the $i N O S$ gene to a radiationinduced promoter to form gene circuit, thereby increasing the duration and the abundance of gene expression.
However, these studies did not solve the problem that the activity of the radiation-induced promoter is reduced in a hypoxic environment. The center of most solid tumors contains a hypoxic environment. In our study, we inserted an HRE upstream of the radiation-induced promoter to reduce the impact of hypoxia on this promoter, as our approach even enhanced the activity of the radiation-induced promoter. In the "Assessment of the expression of IFNG via quantitative-PCR" section, we measured the relative expression levels of IFNG following the transfection of the pYr-ads-8-5HRE-cfosp-iNOS-IFNG or pYr-ads-8-cfospiNOS-IFNG plasmid in a hypoxic environment to evaluate the potentiation of the c-Fos promoter mediated by the HRE. The results showed that the promoter fused to the HRE induced increased expression of IFNG.

Although gene therapy has considerable advantages, the disadvantages of gene transfer must be resolved to successfully implement comprehensive tumor gene therapy. Recently developed nanotechnology has provided a new strategy for resolving the difficulties in the use of gene transfer vectors. ${ }^{49-54}$ The use of nano-vectors for gene transfer has many advantages compared to traditional carriers, including the possibility of repeated injections, the opportunity to effectively extend the response time by slowly releasing the genes, the ability to maintain an effective concentration of the products, and the potential to improve the transfection efficiency and the bioavailability of the products. Many types of magnetic nanoparticles used as gene vectors were prepared in our lab and the transfection efficiency of these nanoparticles has been established. ${ }^{26,27,29}$

Molecular targeted therapy has entered a new era due to the development of molecular biotechnology and the further understanding of the cancer pathogenesis. The specific sites 

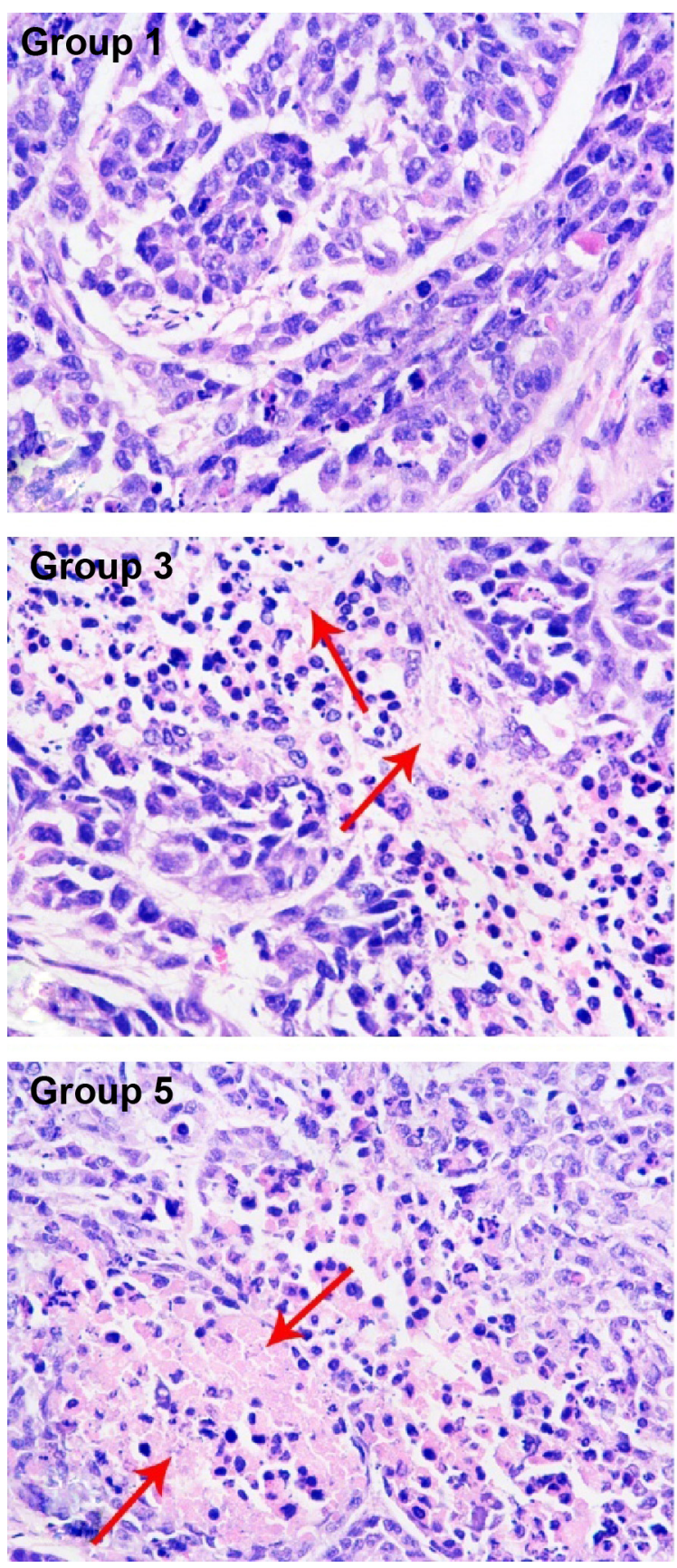
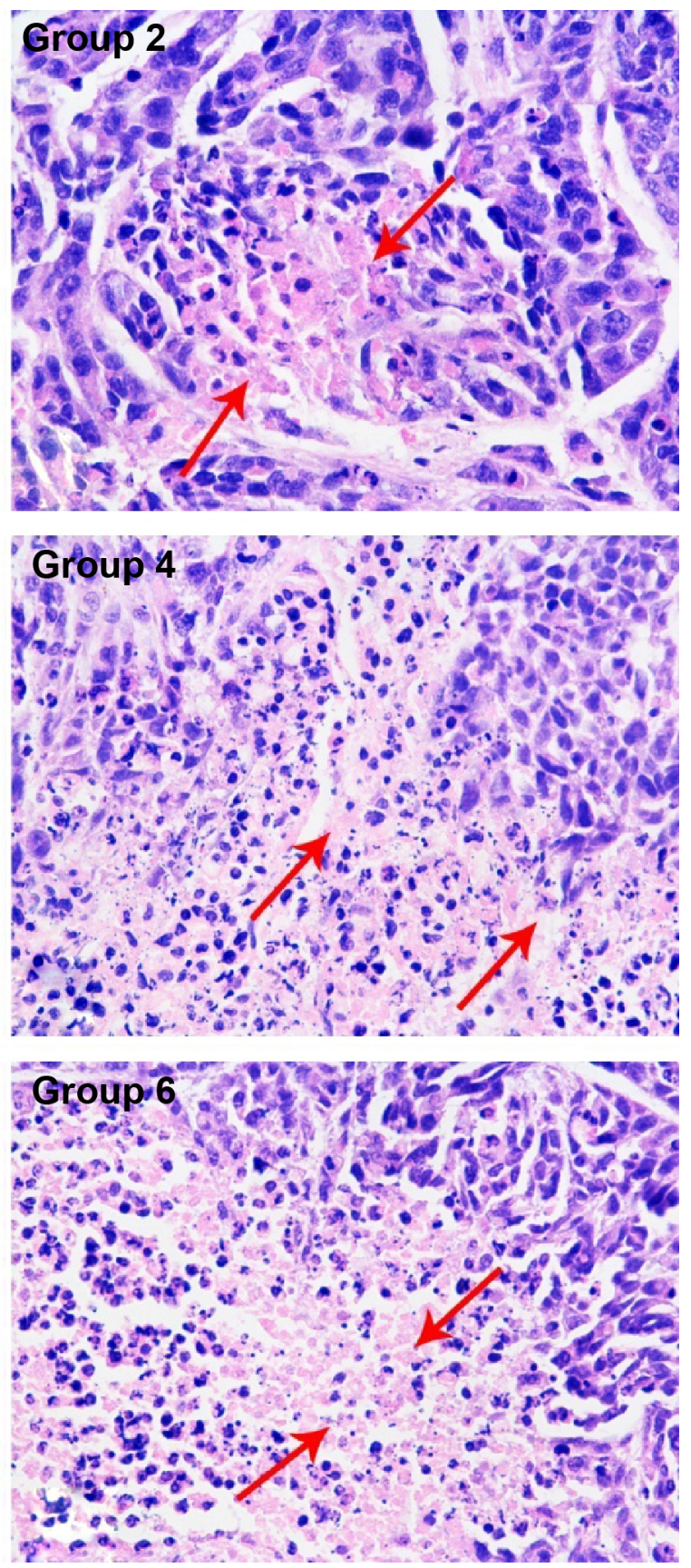

Figure 6 Histological examination of tumor tissues.

Notes: The tumor tissues of the treatment groups displayed different degrees of necrosis (red arrows indicate necrotic tumor tissues). Tumor necrosis was the most extensive in group 6 (the necrotic areas are stained pink). Images of tissues stained with hematoxylin and eosin were captured at 200× magnification. (Group I) negative control, (Group 2) free C225 therapy, (Group 3) radiotherapy, (Group 4) gene therapy combined with radiotherapy, (Group 5) free C225 combined with radiotherapy, and (Group 6) gene-loaded IMANS combined with radiotherapy.

Abbreviation: $\mathrm{C} 225$, cetuximab.

that play a key role in cell growth and proliferation are referred to as target sites. The ideal target sites have high specificity, which indicates that the target sites exhibit little to no expression in normal tissues but display specific expression in tumor tissues. By acting on target sites, tumor cells can be specifically killed while avoiding injury to normal cells. EGFR is overexpressed in $40 \%-50 \%$ of NSCLC cases. Therefore, EGFR is an ideal target gene for lung cancer.
C225, a monoclonal antibody against EGFR, has been used with good curative effect in the clinic.

The therapeutic effect of many monoclonal anticancer antibodies, including $\mathrm{C} 225$, is dependent on the cytotoxic response of the immune reaction induced against cancer cells coated with antigen-bound antibodies. Thus, the important factors to be considered when fusing monoclonal antibodies to cytotoxic drugs or to the surface of nanospheres concern 
the necessity of retaining the primary specific immunoreactivity of the monoclonal antibodies. We fused C225 to MANS to construct IMANS; then, we used IMANS for diagnosis or treatment. ${ }^{55,56}$ In these studies, we have evaluated the immunoreactivity of immunonanospheres and the results showed that $\mathrm{C} 225$ fused to the particles maintain their ability to react with IgG antiserum. Hence, in this study, we adopted this technology to construct gene-loaded IMANS, but we did not reassess the immunoreactivity of gene-loaded IMANS. The transmission electron microscopy images of nanospheres (Figure 3A) showed that the magnetic nanoparticles were successfully incorporated into the matrix of gene-loaded IMANS. This observation indicates that the method for the preparation of gene-loaded IMANS is feasible. We combined nanotechnology with molecular targeted and gene therapies to explore a new treatment model for lung cancer. In our study, in addition to the negative control group, five treatment groups were examined. We compared the difference between each group to verify whether our combination therapy was more effective than any other single treatment. Based on our results, our combination therapy was successful. As shown in Figure 5, we observed that the cytotoxicity to GLC-82 cells in groups 4 (gene therapy combined with radiotherapy) and 5 (C225 combined with radiotherapy) was higher than that in the single treatment groups (the free C225 therapy group and the radiotherapy group). Furthermore, group 6 displayed the highest cytotoxicity. The results of animal experiments followed the same trend. These results showed that our combination therapy was effective. However, many studies remain to be performed. For example, the antitumor mechanism of each treatment included in this combination therapy is uncertain. We will continue to perform more detailed and intensive studies in the future.

\section{Conclusion}

In this study, the 5HRE-cfosp-iNOS-IFNG plasmid was successfully constructed and the expression of this plasmid was validated. Gene-loaded IMANS (C225/HRE-cfosiNOS-IFNG) were prepared and their antitumor effect was demonstrated in vitro and in vivo. The results showed that a combination therapy consisting of radiotherapy, gene therapy, and molecular targeted therapy displays good potential for the treatment of lung cancer.

\section{Acknowledgment}

This project was supported by the National Natural Science Foundation of China (81171452).

\section{Disclosure}

The authors report no conflicts of interest in this work.

\section{References}

1. Ulahannan SV, Brahmer JR. Antiangiogenic agents in combination with chemotherapy in patients with advanced non-small cell lung cancer. Cancer Invest. 2011;29(4):325-337.

2. Jemal A, Bray F, Center MM, et al. Global cancer statistics. CA Cancer J Clin. 2011;61(2):69-90.

3. Ramalingam S, Belani C. Systemic chemotherapy for advanced non-small cell lung cancer: recent advances and future directions. Oncologist. 2008;13(Suppl 1):5-13.

4. Ferlay J, Bray F, Pisani P, et al. GLOBOCAN 2002: cancer incidence, mortality and prevalence worldwide. IARC Cancer Base No 5, version 2.0.Lyon. France: IARC Press; 2004.

5. Anderson WF. Human gene therapy. Nature. 1998;392(6679 Suppl): 25-30.

6. Poulsen TT, Pedersen N, Poulsen HS, et al. Replacement and suicide gene therapy for targeted treatment of lung cancer. Clin Lung Cancer. 2005;6(4):227-236.

7. Wang XY, Croswton JG, White AJ, et al. Interferon-alpha and interferon-gamma modulate Fas-mediated apoptosis in mitomycinC-resistant human Tenon's fibroblasts. Clin Experiment Ophthalmol. 2014;42:529-538

8. Xu B, He YF, Wu XH, et al. Exploration of the correlations between interferon- $\gamma$ in patient serum and HEPACAM in bladder transitional cell carcinoma, and the interferon- $\gamma$ mechanism inhibiting BIU-87 proliferation. J Urol. 2012;188(4):1346-1353.

9. Park SY, Seol JW, Lee YJ, et al. IFN $\gamma$ enhance TRAIL induced apoptosis through IRF-1. Eur J Biochem. 2004;271(21):4222-4228.

10. Stackhouse MA, Buchsbaum DJ. Radiation to control gene expression. Gene Ther. 2000;7(13):1085-1086.

11. Advani S, Chmura SJ, Weichselbaum RR. Radiogenetic therapy: on the interaction of viral therapy and ionizing radiation for improving local control of tumors. Semin Oncol. 1997;24(6):633-638.

12. Weichselbaum RR, Kufe DW, Advani SJ, et al. Molecular targeting of gene therapy and radiotherapy. Acta Oncol. 2001;40(6):735-738.

13. Hasty J, McMillen D, Collins JJ. Engineered gene circuits. Nature. 2002;420:224-230.

14. Maeda YT, Sano M. Regulatory dynamics of synthetic gene networks with positive feedback. J Mol Biol. 2006;359:1107-1124.

15. Ferrell JE Jr. Self perpetuating states in signal transduction: positive feedback, double negative feedback and bistability. Curr Opin Cell Biol. 2002;14:140-148.

16. Dachs GU, Patterson AV, Firth JD, et al. Targeting gene expression to hypoxic tumor cells. Nat Med. 1997;3:515-520.

17. Shibata T, Giaccia AJ, Brown JM. Development of a hypoxia-responsive vector for tumor-specific gene therapy. Gene Ther. 2000;7:403-498.

18. Dash PR, Read ML, Barrett LB, et al. Factors affecting blood clearance and in vivo distribution of polyelectrolyte complexes for gene delivery. Gene Ther. 1999;6(4):643-650.

19. Noguiez-HellinP, Meur MR, Salzmann JL, et al. Plasmoviruses: nonviral/viral vectors for gene therapy. Proc Natl Acad Sci U S A. 1996; 93(9):4175-4180.

20. Scollay R. Gene therapy: a brief overview of the past, present, and future. Ann N Y Acad Sci. 2001;95:26-30.

21. Li X, Luo H, Lin G, Zhou K. Construction of recombinant lentiviral expression vector targeting human Bax inhibitor-1 gene and its expression in NIH3T3 cells. Med Postgra. 2010;232(12):1240-1243.

22. Cohen H, Levy RJ, Gao J, et al. Sustained delivery and expression of DNA encapsulated in polymeric nanoparticles. Gene Ther. 2000; 7(22):1896-1905.

23. Engelhardt JF, Ye X, Doranz B, et al. Ablation of E-A in recombinant adenoviruses improves transgene persistence and decreases inflammatory response in mouse liver. Proc Natl Acad Sci U S A. 1994;91(13): 6196-6200. 
24. Sandhu KK, McIntosh CM, Simard JM, et al. Gold nanoparticles mediated transfection of mammalian cells. Bioconjugate Chem. 2002;13:3-6.

25. Cho WS, Kim S, Han BS. Comparison of gene expression profiles in mice liver following intravenous injection of 4 and $100 \mathrm{~nm}$-sized PEGcoated gold nanoparticles. Toxicol Lett. 2009;191(1):96-102.

26. Tang QS, Zhang DS, Cong XM, Wan ML, Jin LQ. Using thermal energy produced by irradiation of Mn-Zn ferrite magnetic nanoparticles (MZF-NPs) for heat-inducible gene expression. Biomaterials. 2008;29(17):2673-2679.

27. Wang ZY, Wang L, Zhang J, Li YT, Zhang DS. A study on the preparation and characterization of plasmid DNA and drug-containing magnetic nanoliposomes for the treatment of tumors. Int $J$ Nanomedicine. 2011;6:871-875.

28. Connor EE, Mwamuka J, Gole A, et al. Gold nanoparticles are taken up by human cells but do not cause acute cytotoxicity. Small. 2005; 1(3):325-327.

29. Li Y, Liu J, Zhong Y, et al. Biocompatibility of $\mathrm{Fe}_{3} \mathrm{O}_{4} @ \mathrm{Au}$ composite magnetic nanoparticles in vitro and in vivo. Int J Nanomedicine. 2011; 6:2805-2819

30. Yan SY, Zhang DS, Gu N, et al. The preparation and biocompatibility study on $\mathrm{Fe}_{2} \mathrm{O}_{3}$ magnetic nanoparticles used in tumor hyperthermia. Chin J Biomed Eng (English Edition). 2007;16(3):101-110.

31. Jung J, Matsuzaki T, Tatematsu K, et al. Bio-nanocapsule conjugated with liposomes for in vivo pinpoint delivery of various materials. J Control Release. 2008;126(3):255-264.

32. Li Y, Cui HX, Liu Q, Cui JH. The progress of using magnetic nanoparticles as gene vector. Funct Mater. 2010;41(Suppl 2):14-19.

33. Dobson J. Magnetic micro- and nano-particle-based targeting for drug and gene delivery . Nanomedicine. 2006;1(1):31-37.

34. Buka S, Jie S, Shusheng X, Shuli W. Monoclonal antibody against human bladder cancer linked with adriamycin loaded human albumin nanospheres and its cytotoxic effects. Chin J Microbiol Immunol. 1996; 16(1):54.

35. Li Y, Cai M, Liu X, Kuang A, Liang Z. The mechanism of anti-hepatoma effects of adriamycin-loaded immunonanoparticles led by monoclonal antibodies. Chin Pharmacol Bullet. 2001;17(4):463-466.

36. Hirsch F, Scagliotti GV, Langer CJ, Varella-Garcia M, Franklin WA. Epidermal growth factor family of receptors in preneoplasia and lung cancer. Perspectives for targeted therapies. Lung Cancer. 2003;41:S29-S41.

37. Cunningham D, Hummblet Y, Siena VS, et al. Cetuximab monotherapy and cetuximab plus irinotecan in irinotecan-refractory metastatic colorectal cancer. $N$ Engl J Med. 2004;351:337-345.

38. Chen Z, Chen Z. Effects of monoclonal antibody cetuximab on proliferation of non-small cell lung cancer cell lines. Chin J Lung Cancer. 2010;13(8):769-772.

39. Pirazzoli V, Ayeni D, Meador CB, et al. Afatinib plus cetuximab delays resistance compared to single agent erlotinib or afatinib in mouse models of TKI-naïve EGFR L858R-induced lung adenocarcinoma. Clin Cancer Res. 2016;22(2):426-435.

40. Muller BG. Albumin nanospheres as carriers for passive drug targeting: an optimized manufacturing technique. Pharm Res. 1996;13(1): $32-37$.
41. Milde-Langosch K. The Fos family of transcription factors and their role in tumourigenesis. Eur J Cancer. 2005;41(16):2449-2461.

42. Bi JX, Wirth M, Beer C, et al. Dynamic characterization of recombinant Chinese hamster ovary cells containing an inducible c-fos promoter GFP expression system as a biomarker. J Biotechol. 2002;93(3):231-242.

43. Weichselbaum RR, Hallahan ED, Beckett MA, et al. Gene therapy targeted by radiation preferentially radio sensitizes tumors cells. Cancer Res.1994;54:4266-4269.

44. Greco O, Joiner MC, Doleh A. Hypoxia- and radiation-activated Cre/ loxP 'molecular switch' vectors for gene therapy of cancer. Gene Ther. 2006;13:206-215.

45. Du N, Feng K, Luo C, et al. Radioprotective effect of FLT3 ligand expression regulated by Egr-1 regulated element on radiation injury of SCID mice. Exp Hematol. 2003;31(3):191-196.

46. Bogdan C. Nitric oxide and the regulation of gene expression. Trends Cell Biol. 2001;11(2):66-75.

47. Magdalena A, Jolanta, FW. Nitric oxide as a bioactive signalling molecule in plant stress responses. Plant Sci. 2007;172(5):876-887.

48. Worthington J, Robson T, Murray M, et a1. Modification of vascular tone using iNOS under the control of a radiation-inducible promoter. Gene Ther. 2000;7(8):1126-1131.

49. Worthington J, Robson T, Scott S, et al. Evaluation of a synthetic CArG promoter for nitric oxide synthase gene therapy of cancer. Gene Ther. 2005;12(19):1417-1423.

50. Einat CS, Michael C, Dikla G, Sivan K, Nickolay K, Gershon G. Characterization of monocytes-targeted nanocarriers biodistribution in leukocytes in ex vivo and in vivo models. Nano Biomed Eng. 2010; 2:91-99.

51. Edmund A, Kambalapally S, Wilson T, Nicolosi JR. Dextran-based nanocarriers as efficient media delivery vehicles to cell production bioreactors. Nano Biomed Eng. 2010;2:126-132.

52. Huang P, Li Z, Lin J, et al. Photosensitizer-conjugated magnetic nanoparticles for in vivo simultaneous magnetofluorescent imaging and targeting therapy. Biomaterials. 2011;32:3447-3458

53. Gao G, Huang P, Zhang YX, Wang K, Qin W, Cui DX. Gram scale synthesis of superparamagnetic $\mathrm{Fe}_{3} \mathrm{O}_{4}$ nanoparticles and fluid via a facile solvothermal route. CrystEngComm. 2011;13:1782-1785.

54. Shi S, Zhu XC, Guo QF, et al. Self-assembled triblock copolymer mPEG-PCL-gPEI for co-delivery of drug and DNA: synthesis and characterization in vitro. Int J Nanomed. 2012;7:1749-1759.

55. Wang L, An Y, Yuan C, et al. GEM-loaded magnetic albumin nanospheres modified with cetuximab for simultaneous targeting, magnetic resonance imaging, and double-targeted thermochemotherapy of pancreatic cancer cells. Int J Nanomedicine. 2015;10:2507-2519.

56. Hou X, Zhang H, Li H, Zhang D. Magnetic albumin immuno-nanospheres as an efficient gene delivery system for a potential use in lung cancer: preparation, in vitro targeting and biological effect analysis. Drug Target. Epub 2015 Jul 31.
International Journal of Nanomedicine

\section{Publish your work in this journal}

The International Journal of Nanomedicine is an international, peerreviewed journal focusing on the application of nanotechnology in diagnostics, therapeutics, and drug delivery systems throughout the biomedical field. This journal is indexed on PubMed Central, MedLine, CAS, SciSearch ${ }^{\circledR}$, Current Contents ${ }^{\circledR} /$ Clinical Medicine,
Dovepress

Journal Citation Reports/Science Edition, EMBase, Scopus and the Elsevier Bibliographic databases. The manuscript management system is completely online and includes a very quick and fair peer-review system, which is all easy to use. Visit http://www.dovepress.com/ testimonials.php to read real quotes from published authors. 